\title{
ON THE MONODROMY OF A FUNCTION GERM DEFINED ON AN ARRANGEMENT OF HYPERPLANES
}

\author{
MARIA IOACHIM ZAHARIA
}

\section{Introduction}

Let $\mathscr{A}=\left\{H_{1}, \ldots, H_{k}\right\}$ be an arrangement of hyperplanes in $C^{n+1}$ such that $0 \in H_{1} \cap \cdots \cap H_{k}$ and let $\mathscr{L}(\mathscr{A})$ denote the intersection poset of $\mathscr{A}$. Let $f:\left(H_{1} \cup \cdots \cup H_{k}, 0\right) \rightarrow C$ be a germ of a holomorphic function in the origin with the property that the restriction of $f$ to any $X \in \mathscr{L}(\mathscr{A}), X \neq C^{n+1}, X \neq\{0\}$, has an isolated critical point in 0 . It is known that $f$ defines a Milnor fibration (see [2]) and that the Milnor fiber of $f$, denoted by $F$, has the homotopy type of a bouquet of spheres of (real) dimension $n-1$.

Let $h: H_{n-1}(F) \rightarrow H_{n-1}(F)$ be the (algebraic) monodromy and $\Delta(t)=$ $\operatorname{det}(t I-h)$ be its characteristic polynomial. For $X \in \mathscr{L}(\mathscr{A}), X \neq C^{n+1}, X \neq$ $\{0\}$, let $\Delta_{X}(t)$ denote the characteristic polynomial of the monodromy and $\mu\left(\left.f\right|_{X}\right)$ denote the Milnor number of the restriction of $f$ to $X$. If $\{0\} \in \mathscr{L}(\mathscr{A})$ we put $\Delta_{\{0\}}(t)=t-1$ and $\mu\left(\left.f\right|_{\{0\}}\right)=1$. Let $\mu: \mathscr{L}(\mathscr{A}) \rightarrow Z$ be the Möbius function of $\mathscr{L}(\mathscr{A})$.

In this article we shall prove the following theorem (we consider the reduced homology with integer coefficients):

THEOREM 1.1. Under the above conditions, we have:

$$
\Delta(t)=\prod_{X \in \mathscr{L}(\mathscr{A}), X \neq C^{n+1}} \Delta_{X}^{|\mu(X)|}(t) .
$$

In Section 3 we shall use Theorem 1.1 to obtain formulas for the $\zeta$ function of the monodromy, the Lefschetz number of $f$ and the Milnor number of $f$, depending on the similar objects of the restriction of $f$ to the linear spaces $X \in \mathscr{L}(\mathscr{A})$ and on the values of the Möbius function of $\mathscr{L}(\mathscr{A})$.

These results answer question raised by Professor D. Siersma to whom I would like to thank. I am also grateful to the referee for useful suggestions.

We shall remind some facts on arrangements of hyperplanes in a vector space, which we shall need in the proof of Theorem 1.1. These facts can be found in [4].

Recelved October 12, 1998; revised April 1, 1999 
Let $\mathscr{A}=\left\{H_{1}, \ldots, H_{k}\right\}$ be an arrangement of hyperplanes in a vector space $V$ such that $0 \in T(\mathscr{A})=H_{1} \cap \cdots \cap H_{k}$. Let $\mathscr{L}=\mathscr{L}(\mathscr{A})$ be the intersection poset of $\mathscr{A}$ :

$$
\mathscr{L}(\mathscr{A})=\{V\} \cup\left\{W \mid \exists\left\{i_{1}, \ldots, i_{p}\right\} \subseteq\{1, \ldots, k\} \text { such that } W=H_{l_{1}} \cap \cdots \cap H_{l_{p}}\right\} .
$$

On $\mathscr{L}$ a partial order is defined by reverse inclusion: $X \leq Y \Leftrightarrow Y \subseteq X$.

Definition 1.2. The Möbius function $\mu_{\mathscr{A}}: \mathscr{L} \times \mathscr{L} \rightarrow Z$ is defined by

$$
\begin{gathered}
\mu(X, X)=1, \quad \text { if } X \in \mathscr{L}, \\
\sum_{X \leq Z \leq Y} \mu(X, Z)=0, \quad \text { if } X, Y, Z \in \mathscr{L} \text { and } X<Y, \\
\mu(X, Y)=0, \text { otherwise. }
\end{gathered}
$$

Definition 1.3. For $X \in \mathscr{L}$, we define $\mu(X)=\mu(V, X), \mathscr{A}_{X}=\{H \in \mathscr{A} \mid$ $X \subseteq H\}$ and $r(X)=\operatorname{codim} X=\operatorname{dim} V-\operatorname{dim} X$. We denote $\mu(\mathscr{A})=\mu(T(\mathscr{A}))$.

Remark 1.4. It is well-known, see for instance [4], that $\sum_{X \in \mathscr{L}} \mu(X)=0$ and that for $X \in \mathscr{L}$, we have $\mu(X)=(-1)^{r(X)}|\mu(X)|=\mu\left(\mathscr{A}_{X}\right)$.

\section{Proof of Theorem 1.1}

We prove Theorem 1.1 by double induction on the number of hyperplanes in the arrangement, $k$, and the dimension of the base space, $n+1$.

For $k=1$ and any $n$, we have one hyperplane $H$ in $C^{n+1}$ so $\operatorname{dim} H=n$ and we work in fact with $\left.f\right|_{H}$. We have $\Delta(t)=\Delta_{H}(t)$.

For $n=1$ and any $k$ we have $k$ (complex) lines, $H_{1}, \ldots, H_{k}$ in $C^{2}$ and $H_{1} \cap \cdots \cap H_{k}=\{0\}$. The Milnor fiber $F$ of $f$ is a finite set of points and consequently the only nonzero homology group is $H_{0}(F)$. We have $\mathscr{L}(\mathscr{A})=$ $\left\{\boldsymbol{C}^{2}, H_{1}, \ldots, H_{k},\{0\}\right\}$ with $\mu\left(\boldsymbol{C}^{2}\right)=1, \quad \mu\left(H_{l}\right)=-1, \quad \forall i \in\{1, \ldots, k\}, \mu(\{0\})=$ $k-1$. The formula to prove is

$$
\Delta(t)=(t-1)^{k-1} \prod_{l=1}^{k} \Delta_{H_{l}}(t) .
$$

For $k=2$ : Let $F_{1}=F \cap H_{1}$ be the Milnor fiber of the restriction $\left.f\right|_{H_{1}}$ and let $F_{2}=F \cap H_{2}$ be the Milnor fiber of the restriction $\left.f\right|_{H_{2}}$. Then $F_{1}$ consists of $\mu\left(\left.f\right|_{H_{1}}\right)+1$ points, say $x_{0}, x_{1}, \ldots, x_{\mu\left(\left.f\right|_{H_{1}}\right)}$, and $F_{2}$ consists of $\mu\left(\left.f\right|_{H_{2}}\right)+1$ points, say $y_{0}, y_{1}, \ldots, y_{\mu\left(\left.f\right|_{H_{2}}\right)}$. Since $F_{1} \cap F_{2}=\emptyset, F=F_{1} \cup F_{2}$ consists of $\mu\left(\left.f\right|_{H_{1}}\right)+$ $\mu\left(\left.f\right|_{H_{2}}\right)+2$ points and $\operatorname{dim} H_{0}(F)=\mu\left(\left.f\right|_{H_{1}}\right)+\mu\left(\left.f\right|_{H_{2}}\right)+1$. Let us consider the Mayer-Vietoris sequence for $F=F_{1} \cup F_{2}$ :

$$
0 \rightarrow H_{0}\left(F_{1}\right) \oplus H_{0}\left(F_{2}\right) \rightarrow H_{0}(F) .
$$

A basis in $H_{0}\left(F_{1}\right)$ is $\left\{x_{0}-x_{j} \mid j=1,2, \ldots, \mu\left(\left.f\right|_{H_{1}}\right)\right\}$. 
A basis in $H_{0}\left(F_{2}\right)$ is $\left\{y_{0}-y_{i} \mid i=1,2, \ldots, \mu\left(\left.f\right|_{H_{2}}\right)\right\}$.

A basis in $H_{0}(F)$ is

$\left\{x_{0}-x_{J}, y_{0}-y_{i}, x_{0}-y_{0} \mid j=1,2, \ldots, \mu\left(\left.f\right|_{H_{1}}\right)\right.$ and $\left.i=1,2, \ldots, \mu\left(\left.f\right|_{H_{2}}\right)\right\}$.

By [2], the monodromy respects the stratification. Thus, if $h\left(x_{0}\right)=x_{J}$ and $h\left(y_{0}\right)=y_{i}$ for some $j \in\left\{0,1, \ldots, \mu\left(\left.f\right|_{H_{1}}\right)\right\}$ and some $i \in\left\{0,1, \ldots, \mu\left(\left.f\right|_{H_{2}}\right)\right\}$, then

$$
h\left(x_{0}-y_{0}\right)=x_{J}-y_{i}=-x_{0}+x_{J}+y_{0}-y_{i}+x_{0}-y_{0} .
$$

Thus, the matrix of the monodromy $h: H_{0}(F) \rightarrow H_{0}(F)$ in the above basis is

$$
\left(\begin{array}{ccc}
\begin{array}{c}
\text { Matrix of the } \\
\text { monodromy } \\
\text { of }\left.f\right|_{H_{1}}
\end{array} & 0 & 0 \\
0 & \begin{array}{c}
\text { Matrix of the } \\
\text { monodromy } \\
\text { of }\left.f\right|_{H_{2}}
\end{array} & 0 \\
& * & 1
\end{array}\right) .
$$

Consequently, the characteristic polynomial of $h$ is

$$
\Delta(t)=\Delta_{H_{1}}(t) \cdot \Delta_{H_{2}}(t) \cdot(t-1) .
$$

The induction step $k \mapsto k+1$ : The induction hypothesis is: for $k$ lines in $C^{2}$ the characteristic polynomial of the monodromy is

$$
\Delta(t)=\Delta_{H_{1}}(t) \cdots \Delta_{H_{k}}(t) \cdot(t-1)^{k-1} .
$$

Let $H_{1}, \ldots, H_{k+1}$ be $k+1$ lines in $C^{2}$ such that $H_{1} \cap \cdots \cap H_{k+1}=\{0\}$. The sets of points representing the Milnor fibres of the restrictions $\left.f\right|_{H_{l}}$ do not intersect, hence

$$
\operatorname{dim} H_{0}(F)=\sum_{i=1}^{k+1} \mu\left(\left.f\right|_{H_{l}}\right)+k .
$$

We put $F_{1}=F \cap\left(H_{1} \cup \ldots \cup H_{k}\right)$ and $F_{2}=F \cap H_{k+1}$ and we note that

$$
\operatorname{dim} H_{0}\left(F_{1}\right)=\sum_{l=1}^{k} \mu\left(\left.f\right|_{H_{i}}\right)+(k-1) .
$$

If we fix basis in $H_{0}\left(F_{1}\right)$ and $H_{0}\left(F_{2}\right)$ we can get a basis in $H_{0}(F)$ in the same way we did in the case $k=2$ and, like there, we get

$$
\Delta(t)=\Delta_{H_{k+1}}(t) \cdot(t-1) \cdot(t-1)^{k-1} \prod_{i=1}^{k} \Delta_{H_{i}}(t)=(t-1)^{k} \prod_{l=1}^{k+1} \Delta_{H_{i}}(t) .
$$

Let us consider now that Theorem 1.1 is true for any $p$ hyperplanes in a $(m+1)$ dimensional vector subspace of $C^{n+1}$ for $p \leq k$ and $m \leq n$ and let us prove it for $k+1$ hyperplanes in $C^{n+1}$. So consider $H_{1}, \ldots, H_{k+1} \subseteq C^{n+1}$, 
$f:\left(H_{1} \cup \cdots \cup H_{k+1}, 0\right) \rightarrow C$ as before and let $F$ be the Milnor fiber of $f$. We put:

$$
F_{1}=F \cap\left(H_{1} \cup \cdots \cup H_{k}\right) \quad \text { and } \quad F_{2}=F \cap H_{k+1} .
$$

Thus, $F_{1}$ is the Milnor fiber of the restriction $\left.f\right|_{H_{1} \cup \ldots \cup H_{k}}$ and $F_{1} \cap F_{2}$ is the Milnor fiber of the restriction $\left.f\right|_{H_{k+1} \cap\left(H_{1} \cup \ldots \cup H_{k}\right)}$. Let us consider the following monodromies:

$$
\begin{aligned}
& h=\text { the monodromy of } f \\
& h_{1}=\text { the monodromy of }\left.f\right|_{H_{1} \cup \ldots \cup H_{k}} \\
& h_{2}=\text { the monodromy of }\left.f\right|_{H_{k+1}} \\
& h_{12}=\text { the monodromy of }\left.f\right|_{H_{k+1} \cap\left(H_{1} \cup . . \cup H_{k}\right)} .
\end{aligned}
$$

Because the monodromy respects the stratification, the Mayer-Vietoris sequence for $F=F_{1} \cup F_{2}$ gives us the following commutative diagram:

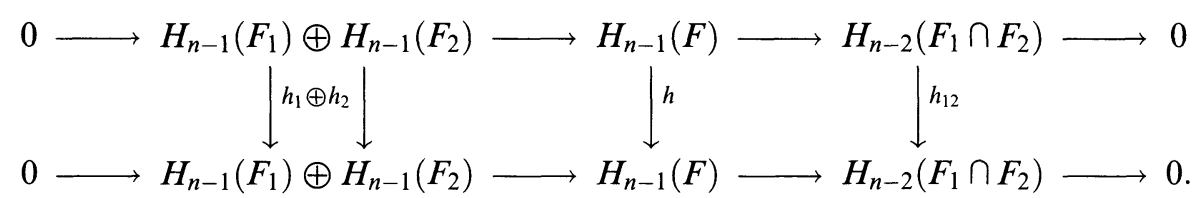

Because the homology groups are free $Z$-modules,

$$
H_{n-1}(F) \simeq H_{n-1}\left(F_{1}\right) \oplus H_{n-1}\left(F_{2}\right) \oplus H_{n-1}\left(F_{1} \cap F_{2}\right)
$$

so there exists a basis in $H_{n-1}(F)$ with respect to which the matrix of the monodromy $h$ consists of cells corresponding to the matrices of $h_{1}, h_{2}$ and $h_{12}$ on the diagonal and zeroes above them (like in the case $k=2$ above). Consequently,

$$
\Delta(t)=\Delta_{H_{k+1}}(t) \cdot \Delta_{2}(t) \cdot \Delta_{12}(t),
$$

where $\Delta_{2}(t)$ and $\Delta_{12}(t)$ are the characteristic polynomials of $h_{2}$ and $h_{12}$.

The induction hypothesis applies for $\left.f\right|_{H_{1} \cup \ldots \cup H_{k}}$, so we have

$$
\Delta_{2}(t)=\prod_{X \in \mathscr{L}^{\prime}, X \neq C^{n+1}} \Delta_{X}^{\left|\mu^{\prime}(X)\right|}(t)
$$

where $\mathscr{A}^{\prime}$ is the arrangement $\left\{H_{1}, \ldots, H_{k}\right\}$ in $C^{n+1}$ and $\mu^{\prime}$ is the Möbius function of $\mathscr{L}^{\prime}:=\mathscr{L}\left(\mathscr{A}^{\prime}\right)$. Next, we can apply the induction hypothesis for $\left.f\right|_{H_{k+1} \cap\left(H_{1} \cap \cdots \cap H_{k}\right)}$ because we have at most $k$ hyperplanes, $H_{1} \cap H_{k+1}, \ldots$, $H_{k} \cap H_{k+1}$, in a $n$-dimensional subspace, $H_{k+1}$, of $C^{n+1}$. So

$$
\Delta_{12}(t)=\prod_{X \in \mathscr{L}^{\prime \prime}, X \neq C^{n+1}} \Delta_{X}^{\left|\mu^{\prime \prime}(X)\right|}(t),
$$

where $\mathscr{A}^{\prime \prime}$ is the arrangement $\left\{H_{1} \cap H_{k+1}, \ldots, H_{k} \cap H_{k+1}\right\}$ in $H_{k+1}$ and $\mu^{\prime \prime}$ is the Möbius function of $\mathscr{L}^{\prime \prime}:=\mathscr{L}\left(\mathscr{A}^{\prime \prime}\right)$. By introducing the relations (2) and (3) in (1), we easily see that the proof of Theorem 1.1 reduces to the proof of the 
following:

$$
\text { For all } X \in \mathscr{L}^{\prime} \cap \mathscr{L}^{\prime \prime} \text {, we have: }|\mu(X)|=\left|\mu^{\prime}(X)\right|+\left|\mu^{\prime \prime}(X)\right| \text {. }
$$

To prove (4), let $X \in \mathscr{L}^{\prime} \cap \mathscr{L}^{\prime \prime}$. Then $X \subseteq H_{k+1}$ and $X=T\left(\mathscr{A}_{X}\right) \in$ $\mathscr{L}\left(\mathscr{A}_{X} \backslash\left\{H_{k+1}\right\}\right)=\mathscr{L}\left(\mathscr{A}_{X}^{\prime}\right)$. Thus, $H_{k+1}$ is not a separator, in the sense of [4], Definition 2.58. And now, point (2) of Corollary 2.59 in [4] gives us that

$$
\left|\mu\left(\mathscr{A}_{X}\right)\right|=\left|\mu\left(\mathscr{A}_{X}^{\prime}\right)\right|+\left|\mu\left(\mathscr{A}_{X}^{\prime \prime}\right)\right| .
$$

By Remark 1.4, relation (5) implies (4). Thus, Theorem 1.1 is proved.

\section{Consequences of Theorem 1.1} we have

Because the degree of the characteristic polynomial is equal to $\operatorname{dim} H_{n-1}(F)$,

Proposition 3.1. Under the conditions in Theorem 1.1, we have

$$
\operatorname{dim} H_{n-1}(F)=\sum_{X \in \mathscr{L}(\mathscr{A}), X \neq C^{n+1}}|\mu(X)| \cdot \mu\left(\left.f\right|_{X}\right) .
$$

Remark 3.2. For another proof of this Proposition, see [8]. In [5] it is obtained a similar result for homogeneous $f$. Note also that the above formula is used in [6].

Remark 3.3. In [7] and [8] we proved a formula to compute the algebraic codimension (when finite) of a function germ $f$ defined on an arrangement $\mathscr{A}=\left\{\left\{x_{1}=0\right\}, \ldots,\left\{x_{p}=0\right\}\right\}$ of coordinate hyperplanes in $\boldsymbol{C}^{n+1}$, when we know the Milnor numbers of its restrictions to the spaces $X \in \mathscr{L}(\mathscr{A})$. It turns out that in this case the algebraic codimension is equal to $\operatorname{dim} H_{n-1}(F)$. We do not know if such a property holds for any arrangement $\mathscr{A}$.

For a function germ $f$ defined on a central arrangement of hyperplanes and having an isolated singularity in 0 , as considered above, the $\zeta$ function of the monodromy is

$$
\zeta_{f}(t)=(1-t)\left[t^{v} \Delta\left(t^{-1}\right)\right]^{(-1)^{n-1}}
$$

where $\Delta$ is the characteristic polynomial of the monodromy and $v$ is the degree of $\Delta$. Using the formulas in Theorem 1.1 and Proposition 3.1, we obtain:

$$
\zeta_{f}(t)=(1-t)\left[\prod_{X \in \mathscr{L}(\mathscr{A}), X \neq C^{n+1}}\left(t^{\mu\left(\left.f\right|_{X}\right)} \Delta_{X}\left(t^{-1}\right)\right)^{|\mu(X)|}\right]^{(-1)^{n-1}} .
$$


But, for any $X \in \mathscr{L}(\mathscr{A}), X \neq\{0\}, X \neq C^{n+1}$, we have

$$
\zeta_{\left.f\right|_{X}}=(1-t)\left[t^{\mu\left(\left.f\right|_{X}\right)} \cdot \Delta_{X}\left(t^{-1}\right)\right]^{(-1)^{\operatorname{dim} X-1}}
$$

so

$$
t^{\mu\left(\left.f\right|_{X}\right)} \cdot \Delta_{X}\left(t^{-1}\right)=\left[(1-t)^{-1} \zeta_{\left.f\right|_{X}}(t)\right]^{(-1)^{\mathrm{dm} X-1}} .
$$

If $\{0\} \in \mathscr{L}(\mathscr{A})$ we put $\zeta_{\left.f\right|_{\{0\}}}(t)=1$. Consequently, by Remark 1.4 we have:

$$
\begin{aligned}
& \zeta_{f}(t)=(1-t)\left[\prod_{X \in \mathscr{L}(\mathscr{A}), X \neq C^{n+1}}\left((1-t)^{-1} \zeta_{\left.f\right|_{X}}(t)\right)^{(-1)^{\operatorname{dim} X-1} \cdot|\mu(X)|}\right]^{(-1)^{n-1}} \\
& =(1-t) \prod_{X \in \mathscr{L}(\mathscr{A}), X \neq C^{n+1}}\left[\left((1-t)^{-1} \zeta_{\left.f\right|_{X}}(t)\right)^{|\mu(X)|}\right]^{(-1)^{\mathrm{d} m X-1-n+1}} \\
& =(1-t) \prod_{X \in \mathscr{L}(\mathscr{A}), X \neq C^{n+1}}\left[(1-t)^{-1} \zeta_{\left.f\right|_{X}}\right]^{|\mu(X)|(-1)^{r(X)-1}} \\
& =(1-t) \prod_{X \in \mathscr{L}(\mathscr{A}), X \neq C^{n+1}}\left[(1-t) \zeta_{\left.f\right|_{X}}^{-1}(t)\right]^{|\mu(X)|(-1)^{r(X)}} \\
& =(1-t) \prod_{X \in \mathscr{L}(\mathscr{A}), X \neq C^{n+1}}(1-t)^{\mu(X)} \zeta_{\left.f\right|_{X}}^{-\mu(X)}(t) \\
& =\prod_{X \in \mathscr{L}(\mathscr{A}), X \neq \boldsymbol{C}^{n+1}} \zeta_{\left.f\right|_{X}}^{-\mu(X)}(t) .
\end{aligned}
$$

Thus, we proved

Proposition 3.4. Under the hypotheses of Theorem 1.1, the $\zeta$ function of the monodromy of $f$ is

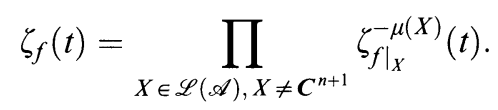

For $X \in \mathscr{L}(\mathscr{A})$, let us denote by $\Lambda_{X}$ the Lefschetz number of the monodromy of $\left.f\right|_{X}$. If $\{0\} \in \mathscr{L}(\mathscr{A})$ we put $\Lambda_{\{0\}}=0$. The Weil inversion formula and Proposition 3.4 imply

Proposition 3.5. Under the conditions in Theorem 1.1, the Lefschetz number of the monodromy is

$$
\Lambda(h)=1+\sum_{X \in \mathscr{L}(\mathscr{A}), X \neq C^{n+1}}\left(1-\Lambda_{X}\right) \mu(X)=-\sum_{X \in \mathscr{L}(\mathscr{A}), X \neq C^{n+1}} \Lambda_{X} \cdot \mu(X) .
$$


Let $(X, 0)$ be the germ of a smooth analytic space, $m_{X, 0}$ the maximal ideal of the local ring $\mathcal{O}_{X, 0}$ and $f$ a function germ defined on $X$. In [1] N. A'Campo proves that for the monodromy $h$ of $f$, we have $\Lambda(h)=0$ if $f \in m_{X, 0}^{2}$ and $\Lambda(h)=$ 1 if $f \in m_{X, 0} \backslash m_{X, 0}^{2}$. Because the subspaces $X \in \mathscr{L}(\mathscr{A})$ are smooth, the formula (6) implies that to compute $\Lambda(h)$ we need only the Lefschetz numbers of those restrictions of $f$ to $X \in \mathscr{L}(\mathscr{A})$ for which $\left.f\right|_{X} \in m_{X, 0}$. Proposition 3.5 and A'Campo's result imply the following

COROllary 3.6. Let $(X, 0)$ be the germ in 0 of a central hyperplane arrangement in $C^{n+1}$, let $m_{X, 0}$ be the maximal ideal of the local ring $\mathcal{O}_{X, 0}$ and let $f$ be a germ of function defined on $(X, 0)$. If $f \in m_{X, 0}^{2}$ then $\Lambda\left(h_{f}\right)=0$.

EXAMPLE 3.7. Let $\mathscr{A}$ be the arrangement of all coordinate hyperplanes in $C^{n+1}$. Its defining ideal is $I=\left(x_{1} \cdots x_{n+1}\right)$ and the maximal ideal $\bar{m}$, of the local ring of this germ of analytic space is the image of the ideal $m=\left(x_{1}, \ldots, x_{n+1}\right)$ in $\mathcal{O}_{n+1} / I$. Let $f \in \bar{m} \backslash \bar{m}^{2}$ be a function germ defined on $\mathscr{A}$. Then, by [7], Proposition 4.1, we have: Either $f$ is $\mathscr{R}$-equivalent to $\overline{x_{1}+\cdots+x_{n+1}}$, in which case

$$
\Lambda\left(h_{f}\right)=1+\mu(\{0\})=1+(-1)^{n+1},
$$

or $f$ is $\mathscr{R}$-equivalent to $x_{1}+\cdots+x_{k}+h\left(x_{k+1}, \ldots, x_{n+1}\right)$ for some $k \in\{1, \ldots, n\}$ and $h$ with $j^{1} h=0$. In this situation we identify $\left\{\left(x_{1}, \ldots, x_{n+1}\right) \in C^{n+1}\right) \mid$ $\left.x_{1}=\cdots=x_{k}=0\right\}$ with $C^{n-k+1}$. The elements $X \in \mathscr{L}(\mathscr{A})$ which intervene in the computation of the Lefschetz number of the monodromy of $f$ are in fact the elements of the intersection poset of the arrangement of all coordinate hyperplanes in $C^{n-k+1}$. Let us denote the set of these elements by $\mathscr{L}_{k}$. For $X \in \mathscr{L}_{k}$, the value of $\mu(X)$ is equal to the value of the Möbius function of the arrangement of all coordinate hyperplanes in $C^{n-k+1}$. Using [4], Proposition 2.44, we get

$$
\Lambda\left(h_{f}\right)=1+\sum_{X \in \mathscr{L}_{k}} \mu(X)=1 .
$$

Thus, in this example the Lefschetz number of the monodromy of $f$ can take the values 0,1 or 2 .

\section{REFERENCES}

[1] N. A'CAMPo, Le nombre de Lefschetz d'une monodromie, Indag. Math., 35 (1973), 113-118.

[2] Lê, D. T., Complex analytic functions with isolated singularities, J. Algebraic Geom., 1 (1992), 83-100.

[3] J. Milnor, Singular points of complex hypersurfaces, Ann. Math. Stud., 61, Princeton, 1968.

[4] P OrliK, H. TeraO, Arrangements of hyperplanes, Grundelheren der Mathematischen Wissenschriften, 300, Sprınger-Verlag, 1992.

[ 5] P. Orlik, H. Terao, Arrangements and Milnor fibers, Math. Ann., 301 (1995), 211-235.

[6] A. ZaHARIA, On the bifurcation set of a polynomial function and Newton boundary, II, Koda1 Math. J., 19 (1996), 218-233. 
[7] M. I. Zaharia, Function germs defined on the arrangement of coordinate hyperplanes, Stud. Cerc. Mat., 47 (1995), 271-282.

[ 8 ] M. I. ZAHARIA, The homology of the Milnor fiber of a function germ defined on an arrangement of hyperplanes, Saltama Math. J., 15 (1997), 1-8.

Marı_Zaharı@@manulife.com 